\title{
MEASURE AND DIMENSION OF SUMS AND PRODUCTS
}

\author{
KYLE HAMBROOK AND KRYSTAL TAYLOR
}

\begin{abstract}
We investigate the Lebesgue measure, Hausdorff dimension, and Fourier dimension of sets of the form $R Y+Z$, where $R \subseteq(0, \infty)$ and $Y, Z \subseteq \mathbb{R}^{d}$. We prove a theorem on the Lebesgue measure and Hausdorff dimension of $R Y+Z$; The theorem is a generalized variant of some theorems of Wolff and Oberlin in which $Y$ is the unit sphere, but its proof is much simpler. We also prove a deeper existence theorem: For each $\alpha \in[0,1]$ and for each nonempty compact set $R \subseteq(0, \infty)$, there exists a compact set $Y \subseteq[1,2]$ such that $\operatorname{dim}_{F}(Y)=\operatorname{dim}_{H}(Y)=\overline{\operatorname{dim}_{M}}(Y)=\alpha$ and $\operatorname{dim}_{F}(R Y) \geq \min \left\{1, \operatorname{dim}_{F}(R)+\right.$ $\left.\operatorname{dim}_{F}(Y)\right\}$. This theorem verifies a weak form of a more general conjecture, and it can be used to produce new Salem sets from old ones.
\end{abstract}

\section{INTRODUCTION}

In this paper, we study the Lebesgue measure, Hausdorff dimension, and Fourier dimension of sets in $\mathbb{R}^{d}$ of the form

$$
R Y+Z=\bigcup_{(r, z) \in R \times Z}(r Y+z)=\{r y+z: r \in R, y \in Y, z \in Z\} .
$$

where $R \subseteq(0, \infty)$ and $Y, Z \subseteq \mathbb{R}^{d}$ are non-empty sets.

This problem has been considered before when $Y$ is a smooth surface with nonvanishing curvature; see [1, 12, 16, 18, 19, 20, 21, 22, 23, 24, In each of these references, the non-vanishing curvature assumption is an essential ingredient, as it implies $Y$ supports a Borel probability measure whose Fourier transform decays at $\infty$. Our aim is to understand what happens when $Y$ is an arbitrary set. Fourier decay of measures on $Y$ will turn out to play an important role.

We assume throughout that the sets $R, Y$, and $Z$ are compact in order to guarantee that the set $R Y+Z$ is Borel measurable. We use $\mathcal{L}_{d}(A), \operatorname{dim}_{H}(A), \operatorname{dim}_{F}(A)$, and $\overline{\operatorname{dim}}_{M}(A)$, respectively, to denote the $d$-dimensional Lebesgue measure, Hausdorff dimension, Fourier dimension, and upper Minkowski (or box-counting) dimension of a set $A \subseteq \mathbb{R}^{d}$. Definitions and basic properties of these dimensions are given in Section 1.2 The expression $a \lesssim b$ means $a \leq C b$ for some positive constant $C$ whose precise value is irrelevant in the context. The expression $a \approx b$ means $a \lesssim b$ and $b \lesssim a$.

1.1. Main Results. This paper has two main results: Theorem 1.1 and Theorem 1.6. The first motivates the second.

Theorem 1.1. Let $R \subseteq(0, \infty)$ and $Y, Z \subseteq \mathbb{R}^{d}$ be non-empty compact sets. Let $\delta$ be the maximum of $\operatorname{dim}_{F}(R Y)+\operatorname{dim}_{H}(Z)$ and $\operatorname{dim}_{H}(R Y)+\operatorname{dim}_{F}(Z)$.

2010 Mathematics Subject Classification. Primary 28A78, 28A80, 42A38, 42B10.

Key words and phrases. Hausdorff dimension, Fourier dimension, Minkowski sum, Minkowski product, fractals.

We thank Alex Iosevich and Izabella Łaba for their helpful comments. 
(a) If $\delta>d$, then $\mathcal{L}_{d}(R Y+Z)>0$

(b) If $\delta \leq d$, then $\operatorname{dim}_{H}(R Y+Z) \geq \delta$.

This theorem makes precise the intuition that the Hausdorff dimension of $R Y+Z$ will be at least the "total" dimension of $R, Y$, and $Z$, and that $R Y+Z$ will have positive Lebesgue measure whenever the "total" dimension exceeds the ambient dimension $d$. It is a generalized variant of Theorems of Wolff [23, 24] and Oberlin 18, 19, which are restricted to the special case where $Y$ is the unit sphere in $\mathbb{R}^{d}$. A thorough discussion of Theorem 1.1 is delayed until Section 1.4. We turn now to motivate our second main result: Theorem 1.6 .

In light of Theorem 1.1 lower bounds on $\operatorname{dim}_{F}(R Y)$ are important. The following proposition gives two general bounds. Part (a) is immediate from the definition of Fourier dimension. Part (b) is essentially Theorem 7 of Bourgain 2 .

\section{Proposition 1.2.}

(a) If $R \subseteq \mathbb{R}$ contains a non-zero point and $Y \subseteq \mathbb{R}^{d}$, then $R Y$ contains a dilate of $Y$, hence $\operatorname{dim}_{F}(R Y) \geq \operatorname{dim}_{F}(Y)$.

(b) If $R$ and $Y$ are non-empty compact subsets of $(0, \infty)$, then $\operatorname{dim}_{F}(R Y) \geq$ $\operatorname{dim}_{H}(R)+\operatorname{dim}_{H}(Y)-1$.

One might hope for the following estimate, at least for some specific sets $Y$ :

$$
\operatorname{dim}_{F}(R Y) \geq \min \left\{d, \operatorname{dim}_{F}(R)+\operatorname{dim}_{F}(Y)\right\} .
$$

As the following example shows, (1.1) does not hold in general.

Example 1.3. Any subset of a (d-1)-dimensional linear subspace of $\mathbb{R}^{d}$ has Fourier dimension 0 (see, e.g., [15, p.41], 25, p.64]). Let $Y$ be such a subset. Assume also that $Y$ is compact. Let $R \subseteq \mathbb{R}$ be a compact set with $\operatorname{dim}_{F}(R)>0$. Then $R Y$ is of the same form as $Y$, and so $\operatorname{dim}_{F}(R Y)=\operatorname{dim}_{F}(Y)=0<\operatorname{dim}_{F}(R)$.

However, if $Y$ equals $S_{d-1}$ (the $(d-1)$-dimensional unit sphere in $\left.\mathbb{R}^{d}\right)$, then (1.1) does hold:

Proposition 1.4. For every non-empty compact set $R \subseteq(0, \infty)$,

$$
\operatorname{dim}_{F}\left(R S_{d-1}\right) \geq \operatorname{dim}_{F}(R)+\operatorname{dim}_{F}\left(S_{d-1}\right) .
$$

Note that $\operatorname{dim}_{F}\left(S_{d-1}\right)=\operatorname{dim}_{H}\left(S_{d-1}\right)=\overline{\operatorname{dim}_{M}}\left(S_{d-1}\right)=d-1$ (see, e.g., 14, 25). We wonder if there are other sets in $\mathbb{R}^{d}$ like the sphere. More precisely, we wonder if the following conjecture is true.

Conjecture 1.5. For every $\alpha \in[0, d]$, there exists a compact set $Y \subseteq \mathbb{R}^{d}$ such that $\operatorname{dim}_{F}(Y)=\operatorname{dim}_{H}(Y)=\overline{\operatorname{dim}_{M}}(Y)=\alpha$ and such that, for every non-empty compact set $R \subseteq(0, \infty)$, (1.1) holds.

We are not able to prove this conjecture. However, if we restrict ourselves to dimension $d=1$ and allow $Y$ to depend on $R$, we are able to prove the following weaker version of the conjecture, which is the second main result of this paper.

Theorem 1.6. For every $\alpha \in[0,1]$ and every non-empty compact set $R \subseteq(0, \infty)$, there exists a compact set $Y \subseteq[1,2]$ such that $\operatorname{dim}_{F}(Y)=\operatorname{dim}_{H}(Y)=\overline{\operatorname{dim}_{M}}(Y)=$ $\alpha$ and (1.1) holds. 
The proof of Theorem 1.6 is inspired by a construction of Salem sets due to Eaba and Pramanik [11. See also [3, 5, 6, 7, where generalizations of the Eaba-Pramanik construction were used to show the sharpness of fractal Fourier restriction theorems.

As an immediate consequence of Theorems 1.1 and 1.6, we have:

Corollary 1.7. Suppose $Y, Z \subseteq \mathbb{R}$ are compact sets such that $Y$ contains a non-zero point and $\operatorname{dim}_{F}(Y)+\operatorname{dim}_{H}(Z)>0$. Let $\alpha \in[0,1]$ such that $\operatorname{dim}_{F}(Y)+\operatorname{dim}_{H}(Z)>$ $1-\alpha$. Then there exists a compact set $R \subseteq(0, \infty)$ such that $\operatorname{dim}_{H}(R)=\operatorname{dim}_{F}(R)=$ $\alpha$ and $R Y+Z$ has positive Lebesgue measure.

The proofs of Theorem 1.1 Proposition 1.4, and Theorem 1.6 are given in Sections 2, 3, and 4, respectively. The following construction will be used in all the proofs.

Definition 1.8. Given a Borel measure $\mu$ on $\mathbb{R}$ and a Borel measure $\nu$ on $\mathbb{R}^{d}$, we define the Borel measure $\mu \cdot \nu$ on $\mathbb{R}^{d}$ by

$$
\int_{\mathbb{R}^{d}} f(z) d(\mu \cdot \nu)(z)=\int_{\mathbb{R}^{d}} \int_{\mathbb{R}} f(r y) d \mu(r) d \nu(y) .
$$

It is readily verified that $(\mu \cdot \nu)\left(\mathbb{R}^{d}\right)=\mu(\mathbb{R}) \nu\left(\mathbb{R}^{d}\right)$ and $\operatorname{supp}(\mu \cdot \nu)=\operatorname{supp}(\mu) \operatorname{supp}(\nu)$.

1.2. Dimensions. In this section, we state the definitions and necessary properties of Hausdorff, Fourier, and upper Minkowski dimension. For more details, see [4, 14, 25. The support of a Borel measure $\mu$ on $\mathbb{R}^{d}$, denoted $\operatorname{supp}(\mu)$, is the smallest closed set $F$ with $\mu\left(\mathbb{R}^{d} \backslash F\right)=0$. For each $A \subseteq \mathbb{R}^{d}$, let $\mathcal{M}(A)$ be the set of all non-zero finite Borel measures on $\mathbb{R}^{d}$ with compact support contained in $A$. For every $\mu \in \mathcal{M}\left(\mathbb{R}^{d}\right)$ and $0<s<d$, the $s$-energy of $\mu$ is

$$
I_{s}(\mu)=\int_{\mathbb{R}^{d}} \int_{\mathbb{R}^{d}}|x-y|^{-s} d \mu(x) d \mu(y)=c(d, s) \int_{\mathbb{R}^{d}}|\widehat{\mu}(\xi)|^{2}|\xi|^{s-d} d \xi,
$$

where $c(d, s)=\pi^{s-d / 2} \Gamma((d-s) / 2) / \Gamma(s / 2)$. There are numerous equivalent definitions of Hausdorff dimension. For our purposes, the following is most convenient: The Hausdorff dimension of a Borel set $A \subseteq \mathbb{R}^{d}$ is

$$
\operatorname{dim}_{H}(A)=\sup \left\{0<s<d: I_{s}(\mu)<\infty \text { for some } \mu \in \mathcal{M}(A)\right\} .
$$

The Fourier dimension of a Borel set $A \subseteq \mathbb{R}^{d}$ is

$$
\operatorname{dim}_{F}(A)=\sup \left\{0<s<d: \sup _{\xi \in \mathbb{R}^{d}}|\widehat{\mu}(\xi)|^{2}|\xi|^{s}<\infty \text { for some } \mu \in \mathcal{M}(A)\right\} \text {. }
$$

The Fourier dimension of a measure $\mu \in \mathcal{M}\left(\mathbb{R}^{d}\right)$ is

$$
\operatorname{dim}_{F}(\mu)=\sup \left\{0<s<d: \sup _{\xi \in \mathbb{R}^{d}}|\widehat{\mu}(\xi)|^{2}|\xi|^{s}\right\} .
$$

The upper Minkowski dimension of a non-empty bounded set $A \subseteq \mathbb{R}^{d}$ is

$$
\overline{\operatorname{dim}}_{M}(A)=\limsup _{\epsilon \rightarrow 0^{+}} \frac{\log N(A, \epsilon)}{\log (1 / \epsilon)} .
$$

where $N(A, \epsilon)$ is the smallest number of $\epsilon$-balls needed to cover $A$.

Lemma 1.9. Let $A$ and $B$ be non-empty bounded Borel subsets of $\mathbb{R}^{d}$.

(a) If $f: A \rightarrow \mathbb{R}^{n}$ is a Lipschitz map, then $\operatorname{dim}_{H}(f(A)) \leq \operatorname{dim}_{H}(A)$. 
(b) $\operatorname{dim}_{F}(A) \leq \operatorname{dim}_{H}(A) \leq \overline{\operatorname{dim}}_{M}(A)$.

(c) $\operatorname{dim}_{H}(A)+\operatorname{dim}_{H}(B) \leq \operatorname{dim}_{H}(A \times B) \leq \operatorname{dim}_{H}(A)+\overline{\operatorname{dim}_{M}}(B)$.

(d) If there is a $\mu \in \mathcal{M}(A)$ and positive numbers $r_{0}$ and s such that

$$
\mu(B(x, r)) \approx r^{s} \quad \text { for all } x \in A, 0<r \leq r_{0},
$$

then $\operatorname{dim}_{H}(A)=\overline{\operatorname{dim}_{M}}(A)=s$.

1.3. Reverse of (1.1) and Salem Sets. In this section, we find sufficient conditions for the reverse of inequality (1.1) and for $R Y$ to be a Salem set. A Borel set $A \subseteq \mathbb{R}^{d}$ is called Salem if $\operatorname{dim}_{F}(A)=\operatorname{dim}_{H}(A)$. See [11, 13, 15, 17, for some illustrations of the construction of Salem sets and their usefulness in harmonic analysis.

Let $R \subseteq \mathbb{R}$ and $Y \subseteq \mathbb{R}^{d}$ be non-empty compact sets. By the definition of Fourier dimension, $\operatorname{dim}_{F}(R Y) \leq d$. By parts (a),(b),(c) of Lemma 1.9 and because the map $f(x, y)=x y$ is Lipschitz on bounded sets,

$$
\operatorname{dim}_{F}(R Y) \leq \operatorname{dim}_{H}(R Y) \leq \operatorname{dim}_{H}(R \times Y) \leq \operatorname{dim}_{H}(R)+\overline{\operatorname{dim}_{M}}(Y) .
$$

From this inequality, the following observations are immediate:

(i) If $R$ is a Salem set and $Y$ satisfies $\operatorname{dim}_{F}(Y)=\operatorname{dim}_{H}(Y)=\overline{\operatorname{dim}_{M}}(Y)$, then the reverse of (1.1) holds, i.e.,

$$
\operatorname{dim}_{F}(R Y) \leq \min \left\{d, \operatorname{dim}_{F}(R)+\operatorname{dim}_{F}(Y)\right\} .
$$

(ii) If, in addition to the hypotheses of (i), (1.1) holds, then $R Y$ is a Salem set.

The hypotheses of (i) and (ii) are satisfied if, for example, $Y$ is the unit sphere $S_{d-1}$ or a set furnished by Theorem 1.6. The construction of Salem sets is generally non-trivial; (ii) gives us a way to produce new Salem sets from old ones.

1.4. Discussion of Theorem 1.1. Let $S_{d-1}$ denote the $(d-1)$-dimensional unit sphere in $\mathbb{R}^{d}$. Theorem 1.1 is a generalized variant of the following theorems of Wolff and Oberlin.

Theorem 1.10 (Wolff, Oberlin). Let $K \subseteq(0, \infty) \times \mathbb{R}^{d}$ be a non-empty compact set. If $\operatorname{dim}_{H}(K)>1$, then

$$
\mathcal{L}_{d}\left(\bigcup_{(r, z) \in K}\left(r S_{d-1}+z\right)\right)>0 .
$$

Theorem 1.11 (Wolff). Let $K \subseteq(0, \infty) \times \mathbb{R}^{d}$ be a non-empty compact set. Let $C \subseteq \mathbb{R}^{d}$ be the set of centers of the spheres $\left\{r S_{d-1}+z:(r, z) \in K\right\}$, i.e., $C=$ $\{z:(r, z) \in K$ for some $r\}$. If $\operatorname{dim}_{H}(K) \leq 1$ and $\operatorname{dim}_{H}(C) \leq 1$, then

$$
\operatorname{dim}_{H}\left(\bigcup_{(r, z) \in K}\left(r S_{d-1}+z\right)\right) \geq \operatorname{dim}_{H}(C)+d-1 .
$$

Theorem 1.12 (Oberlin). Let $K \subseteq(0, \infty) \times \mathbb{R}^{d}$ be a non-empty compact set. If $\operatorname{dim}_{H}(K) \leq 1$ and $\operatorname{dim}_{H}(K)<(d-1) / 2$, then

$$
\operatorname{dim}_{H}\left(\bigcup_{(r, z) \in K}\left(r S_{d-1}+z\right)\right) \geq \operatorname{dim}_{H}(K)+d-1 .
$$


Theorem 1.10 is due to Wolff [24, Corollary 3] when $d=2$ and Oberlin [18, Corollary 1] when $d \geq 3$. Theorem 1.12 is due to Oberlin [19, Theorem $3_{S}$ ]. Theorem 1.11] is due to Wolff [23, Corollary 5.4] (see also the remark following the proof). In fact, Wolff and Oberlin proved slightly more general results; we have stated special cases to simplify comparison to Theorem 1.1.

Wolff obtained Theorem 1.10 for $d=2$ as a corollary of localized $L^{p}$ estimates on functions with Fourier support near the light cone; the proof involves intricate bounds on circle tangencies. For $d=2$, the proof of Theorem 1.11] is based on an $L^{3}-L^{3}$ circular maximal inequality, whose proof in turn is also based on bounds on circle tangencies. For $d \geq 3$, Theorem 1.11 follows from an an easier $L^{2}-L^{2}$ spherical maximal inequality. Oberlin obtained Theorem 1.10 for $d \geq 3$ and Theorem 1.12 by way of estimates for spherical averaging operators. Mitsis [16 previously proved a special case of Theorem 1.10 with an additional hypothesis on the Hausdorff dimension of the set of centers $C=\{z:(r, z) \in K$ for some $r\}$; the methods are similar to those used by Wolff for Theorem 1.11. An alternative proof of Mitsis' result using the technology of spherical maximal operators in a fractal setting follows as an immediate consequence of the work of Krause, Iosevich, Sawyer, Taylor, and Uriarte-Tuero [10].

In contrast to the proofs of Theorems 1.10, 1.11, 1.12, our proof of Theorem 1.1 is extremely short and uses only elementary Fourier analysis and geometric measure theory. As described in Section 1.1. Theorem 1.1 also leads to the problem of of obtaining lower bounds on the Fourier dimension of the Minkowski product of two sets, which motivates Theorem 1.6

We call Theorem 1.1 a generalized variant of Theorems 1.10, 1.11, 1.12 because Theorem 1.1 allows an arbitrary set $Y$ in place of the sphere $S_{d-1}$. However, Theorem 1.1 is not a true generalization. For one thing, the union $R Y+Z=$ $\bigcup_{(r, z) \in R \times Z}(r Y+Z)$ in Theorem 1.1 is over the Cartesian product $R \times Z$, while the union in Theorems 1.10 1.11 1.12, is over a more general set $K$.

To compare Theorem 1.1 to Theorems 1.10 1.11 1.12 on the same footing, let $Y=S_{d-1}$, let $K=R \times Z$ (where $R \subseteq(0, \infty)$ and $Z \subseteq \mathbb{R}^{d}$ are compact sets), and let $\delta$ be as in Theorem 1.1. Arguing as in Section 1.3. we find

$$
\operatorname{dim}_{H}(K)+d-1=\operatorname{dim}_{H}\left(R \times S_{d-1} \times Z\right) \geq \operatorname{dim}_{H}\left(R S_{d-1}\right)+\operatorname{dim}_{H}(Z) \geq \delta .
$$

From (1.6), we see that the hypothesis of Theorem 1.1(a) $(\delta>d)$ is stronger than the hypothesis of Theorem $1.10\left(\operatorname{dim}_{H}(K)>1\right)$, while the conclusions are the same. Thus Theorem 1.10 is stronger than Theorem 1.1(a). The situation is more complicated when comparing Theorem 1.1(b) to Theorems 1.11and1.12. By (1.6), the hypothesis of Theorem 1.1(b) is weaker than the hypotheses of Theorems 1.11 and 1.12 but the conclusion of Theorem 1.1 (b) is also weaker.

Theorem 1.1(b) fills some gaps in Theorems 1.11 and 1.12. In particular, Theorem 1.12 misses the endpoint case $\operatorname{dim}_{H}(K)=1$ when $d=3$ and misses the interval $1 / 2 \leq \operatorname{dim}_{H}(K) \leq 1$ when $d=2$. Meanwhile, Theorem 1.11 can only establish that $\operatorname{dim}_{H}\left(\bigcup_{(r, z) \in K} r S_{d}+z\right)$ is at least $\operatorname{dim}_{H}(C)+d-1$, rather than at least $\operatorname{dim}_{H}(K)+d-1$. As the next example shows, Theorem 1.1(b) can give superior information in these cases.

Example 1.13. Let $d=3$. Let $A$ be a compact subset of $(0, \infty)$ such that $\operatorname{dim}_{F}(A)=\operatorname{dim}_{H}(A)=\overline{\operatorname{dim}_{M}}(A)=1 / 4$ (Theorem 1.6 furnishes such a set). Let $R=A, Z=A^{3}$, and $K=R \times Z$. By Lemma [1.9(c), $\operatorname{dim}_{H}(Z)=3 / 4$ 
and $\operatorname{dim}_{H}(K)=1$. Since $\operatorname{dim}_{H}(K) \geq(d-1) / 2$, Theorem 1.12 gives no information about the Hausdorff dimension of $\bigcup_{(r, z) \in K}\left(r S_{2}+z\right)$. Since $Z=C=$ $\{z:(r, z) \in K$ for some $r\}$, Theorem 1.11 implies only that the Hausdorff dimension of $\bigcup_{(r, z) \in K}\left(r S_{2}+z\right)$ is at least $2+3 / 4$. However, by Proposition 1.4 $\operatorname{dim}_{F}\left(R S_{2}\right)+$ $\operatorname{dim}_{H}(Z) \geq \operatorname{dim}_{F}(R)+\operatorname{dim}_{F}\left(S_{2}\right)+\operatorname{dim}_{H}(Z)=3$. So Theorem 1.1(b) implies the Hausdorff dimension of $\bigcup_{(r, z) \in K}\left(r S_{2}+z\right)=R S_{2}+Z$ equals 3. (Taking $R=A$ and $Z=A^{2}$ gives a similar example when $d=2$.)

Theorem 1.10 can be generalized to any $(d-1)$-dimensional surface with nonvanishing Gaussian curvature [8. However, there is no hope of replacing the sphere in Theorem 1.10 by an arbitrary $(d-1)$-dimensional set, as the following example shows.

Example 1.14. Let $Y$ be the intersection of a $(d-1)$-dimensional cone through the origin with an open spherical shell centered at the origin (such as the set $\left.\left\{x \in \mathbb{R}^{d}: 1 / 2<|x|<2\right\}\right)$. Then $R Y$ is of the same form whenever $R \subseteq(0, \infty)$ contains a non-zero point. Let $R=[a, b] \subseteq(0, \infty)$ be a compact interval, let $Z \subseteq \mathbb{R}^{d}$ be a compact set with $\operatorname{dim}_{H}(Z) \in(0,1)$, and let $K=R \times Z$. By Lemma 1.9(c), $\operatorname{dim}_{H}(K) \geq \operatorname{dim}_{H}(R)+\operatorname{dim}_{H}(Z)>1$. But $\bigcup_{(r, z) \in K}(r Y+z)=R Y+Z$ has Hausdorff dimension $\leq d-1+\operatorname{dim}_{H}(Z)<d$, hence has $d$-dimensional Lebesgue measure 0 . To see that $\operatorname{dim}_{H}(R Y+Z) \leq d-1+\operatorname{dim}_{H}(Z)$, we first observe that, as $R Y$ is a smooth $(d-1)$-dimensional manifold, its Hausdorff and upper Minkowski dimensions are both equal to $d-1$. (To see this, one can appeal to Lemma 1.9)(d) and take $\mu$ to be the restriction of the $(d-1)$-dimensional Hausdorff measure to $R Y)$. Thus, by Lemma 1.9(c), $\operatorname{dim}_{H}(R Y \times Z)=d-1+\operatorname{dim}_{H}(Z)$. Then, since $f(x, y)=x+y$ is Lipschitz, Lemma1.9(a) implies $\operatorname{dim}_{H}(R Y+Z) \leq d-1+\operatorname{dim}_{H}(Z)$.

\section{Proof of Theorem 1.1}

2.1. Proof of Part (a). Assume $\operatorname{dim}_{F}(R Y)+\operatorname{dim}_{H}(Z) \geq \operatorname{dim}_{H}(R Y)+\operatorname{dim}_{F}(Z)$. The proof in the opposite case is similar. Assume $\operatorname{dim}_{F}(R Y)+\operatorname{dim}_{H}(Z)>d$. Choose $0<\alpha<\operatorname{dim}_{F}(R Y)$ and $0<\beta<\operatorname{dim}_{H}(Z)$ such that $\alpha+\beta=d$. Choose $\mu \in \mathcal{M}(R Y)$ and $\nu \in \mathcal{M}(Z)$ such that $\sup _{\xi \in \mathbb{R}^{d}}|\widehat{\mu}(\xi)|^{2}|\xi|^{\alpha}<\infty$ and $I_{\beta}(\nu)<\infty$. Then $\mu * \nu \in \mathcal{M}(R Y+Z)$ and

$$
\int|\widehat{\mu * \nu}(\xi)|^{2} d \xi=\int|\widehat{\mu}(\xi)|^{2}|\widehat{\nu}(\xi)|^{2} d \xi \lesssim \int|\xi|^{(d-\alpha)-d}|\widehat{\nu}(\xi)|^{2} d \xi=I_{\beta}(\nu)<\infty .
$$

Since $\widehat{\mu * \nu}$ is in $L^{2}$, a standard argument (e.g. see [15, Theorem 3.3]) shows $\mu * \nu$ is absolutely continuous with $L^{2}$ density, hence $\mathcal{L}_{d}(R Y+Z)>0$.

2.2. Proof of Part (b). Assume $\operatorname{dim}_{F}(R Y)+\operatorname{dim}_{H}(Z) \geq \operatorname{dim}_{H}(R Y)+\operatorname{dim}_{F}(Z)$. The proof in the opposite case is similar. Let $s \in(0, d)$ with $\operatorname{dim}_{F}(R Y)+\operatorname{dim}_{H}(Z)>$ s. If either $\operatorname{dim}_{F}(R Y)=0$ or $\operatorname{dim}_{H}(Z)=0$, then the result is immediate; assume otherwise. Choose $0<\alpha<\operatorname{dim}_{F}(R Y)$ and $0<\beta<\operatorname{dim}_{H}(Z)$ such that $\alpha+\beta=s$. Choose $\mu \in \mathcal{M}(R Y)$ and $\nu \in \mathcal{M}(Z)$ such that $\sup _{\xi \in \mathbb{R}^{d}}|\widehat{\mu}(\xi)|^{2}|\xi|^{\alpha}<\infty$ and $I_{\beta}(\nu)<$ $\infty$. Then $\mu * \nu \in \mathcal{M}(R Y+Z)$ and

$$
\begin{aligned}
I_{s}(\mu * \nu) & =\int|\widehat{\mu * \nu}(\xi)|^{2}|\xi|^{s-d} d \xi=\int|\widehat{\mu}(\xi)|^{2}|\widehat{\nu}(\xi)|^{2}|\xi|^{s-d} d \xi \\
& \lesssim \int|\xi|^{(s-\alpha)-d}|\widehat{\nu}(\xi)|^{2} d \xi=I_{\beta}(\nu)<\infty .
\end{aligned}
$$


By (1.2) and our choice of $s, \operatorname{dim}_{H}(R Y+Z) \geq \operatorname{dim}_{F}(R Y)+\operatorname{dim}_{H}(Z)$.

\section{Proof of Proposition 1.4}

Let $\sigma$ be the surface measure on the $(d-1)$-dimensional unit sphere $S_{d-1} \subseteq \mathbb{R}^{d}$. The following asymptotic is well-known (e.g., see [15, 25): For $\xi \in \mathbb{R}^{d}$,

$$
\begin{aligned}
\widehat{\sigma}(\xi) & =2|\xi|^{-(d-1) / 2} \cos \left(2 \pi\left(|\xi|-\frac{d-1}{8}\right)\right)+O\left(|\xi|^{-(d+1) / 2}\right) \\
& =|\xi|^{-(d-1) / 2}\left(c_{d} e^{2 \pi i|\xi|}+\overline{c_{d}} e^{-2 \pi i|\xi|}\right)+O\left(|\xi|^{-(d+1) / 2}\right),
\end{aligned}
$$

where $c_{d}=e^{-\pi i(d-1) / 4}$. Let $R \subseteq(0, \infty)$. Let $\mu \in \mathcal{M}(R)$ be arbitrary. Choose $a, b>0$ such that $\operatorname{supp}(\mu) \subseteq[a, b] \subseteq(0, \infty)$. Define $\mu_{0}$ by $d \mu_{0}(r)=r^{\frac{(d-1)}{2}} d \mu(r)$. Then $\operatorname{supp}\left(\mu_{0}\right)=\operatorname{supp}(\mu), \mu_{0} \in \mathcal{M}(R)$, and $\mu_{0} \cdot \sigma \in \mathcal{M}\left(R S_{d-1}\right)$. Furthermore,

$$
\widehat{\mu_{0} \cdot \sigma}(\xi)=\int_{a}^{b} \widehat{\sigma}(r \xi) d \mu_{0}(r)=|\xi|^{-(d-1) / 2}\left(c_{d} \widehat{\mu}(-|\xi|)+\overline{c_{d}} \widehat{\mu}(|\xi|)\right)+O\left(|\xi|^{-(d+1) / 2}\right)
$$

for all sufficiently large $\xi \in \mathbb{R}^{d}$. Therefore $\operatorname{dim}_{F}\left(R S_{d-1}\right) \geq \operatorname{dim}_{F}\left(\mu_{0} \cdot \sigma\right) \geq$ $\operatorname{dim}_{F}(\mu)+d-1$ and (consequently) $\operatorname{dim}_{F}\left(R S_{d-1}\right) \geq \operatorname{dim}_{F}(R)+d-1$.

\section{Proof of Theorem 1.6}

In light of parts (b) and (d) of Lemma 1.9 and the definition of Fourier dimension, Theorem 1.6 is implied by:

Theorem 4.1. Let $\alpha \in[0,1]$. Let $R$ be a non-empty compact subset of $(0, \infty)$. Let $\nu \in \mathcal{M}(R)$. Then there is a Borel probability measure $\mu$ on $\mathbb{R}$ such that

(a) $\operatorname{supp}(\mu) \subseteq[1,2]$,

(b) $\mu(B(x, r)) \approx r^{\alpha}$ for all $x \in \operatorname{supp}(\mu)$ and $0<r \leq 1$,

(c) $\operatorname{dim}_{F}(\mu) \geq \alpha$,

(d) $\operatorname{dim}_{F}(\mu \cdot \nu) \geq \min \left\{1, \operatorname{dim}_{F}(\mu)+\operatorname{dim}_{F}(\nu)\right\}$.

The following two sections are devoted to proving Theorem 4.1, In the first section, we give a general construction of a measure $\mu$ that satisfies parts (a) and (b) of Theorem 4.1. Nothing in the first section will depend on $\nu$. In the second section, we specialize the construction of $\mu$ to prove parts (c) and (d). The specialization will depend on $\nu$.

Before we begin, we dispense with a trivial case: If $\alpha=0$, then taking $\mu$ to be a point mass gives the desired result. Hereafter, we assume $\alpha \in(0,1]$.

4.1. Proof of Theorem 4.1; General Construction. For every $n \in \mathbb{Z}_{>0}$, we use the notation $[n]=\{0,1, \ldots, n-1\}$ For sequences $\left(t_{j}\right)_{j=1}^{\infty}$ and $\left(n_{j}\right)_{j=1}^{\infty}$ of positive integers, we use the notation $T_{j}=t_{1} \cdots t_{j}$ and $N_{j}=n_{1} \cdots n_{j}$. We also use the empty product convention, so that $T_{0}=N_{0}=1$.

Fix an integer $n_{*} \geq 2$. Fix sequences $\left(t_{j}\right)_{j=1}^{\infty}$ and $\left(n_{j}\right)_{j=1}^{\infty}$ of positive integers such that, for all $j \in \mathbb{Z}_{>0}$, we have $2 \leq n_{j} \leq n_{*}, 1 \leq t_{j} \leq n_{j}$, and $T_{j} \approx N_{j}^{\alpha}$.

We recursively define two families of sets:

$$
\left\{A_{j}: j \in \mathbb{Z}_{\geq 0}\right\} \text { and }\left\{B_{j+1, a}: j \in \mathbb{Z}_{\geq 0}, a \in A_{j}\right\} .
$$

Define $A_{0}=\{1\}$. Assuming that $A_{j}$ has been defined for a fixed $j \in \mathbb{Z}_{\geq 0}$, for each $a \in A_{j}$ choose a set $B_{j+1, a} \subseteq N_{j+1}^{-1}\left[n_{j+1}\right]$ such that $\left|B_{j+1, a}\right|=t_{j+1}$. Later we make a specific choice for the sets $B_{j+1, a}$, but for now the sets $B_{j+1, a}$ are arbitrary. 
Define $A_{j+1}=\bigcup_{a \in A_{j}}\left(a+B_{j+1, a}\right)$. Note that this recursive definition implies that $A_{j} \subseteq[1,2)$ and $\left|A_{j}\right|=T_{j}$ for all $j \in \mathbb{Z}_{\geq 0}$.

Question: What are the sets $A_{j}$ and $B_{j+1, a}$ ? Answer: They are sets of endpoints in the following Cantor set construction. Start with the interval [1,2]. Divide it into $n_{1}$ intervals of length $1 / N_{1}$, keep $t_{1}$ of these intervals, and discard the rest. For each of the kept intervals, we do the following: Divide it into $n_{2}$ intervals of length $1 / N_{2}$, keep $t_{2}$ of these intervals, and discard the rest. This gives, in total, $T_{2}$ intervals of length $1 / N_{2}$. Continuing in this way, at the $j$-th stage we have $T_{j}$ intervals of length $1 / N_{j}$. The set of left endpoints of these intervals is $A_{j}$. For each of these intervals, we do the following: Divide it into $n_{j+1}$ intervals of length $1 / N_{j+1}$, keep $t_{j+1}$ of these intervals, and discard the rest. If $a$ is the left endpoint of the interval we started with, the set of left endpoints of the intervals kept is $a+B_{j+1, a}$. The union of all the sets $a+B_{j+1, a}$ (as $a$ ranges over $A_{j}$ ) is $A_{j+1}$. The Cantor set constructed is

$$
\bigcap_{j=0}^{\infty} \bigcup_{a \in A_{j}}\left[a, a+N_{j}^{-1}\right] .
$$

For each $j \in \mathbb{Z}_{\geq 0}$, define $\mu_{j}$ to be the probability measure whose density with respect to Lebesgue measure on $\mathbb{R}$ is

$$
\mu_{j}=\frac{N_{j}}{T_{j}} \sum_{a \in A_{j}} \mathbf{1}_{\left[a, a+N_{j}^{-1}\right]} .
$$

Note that we have abused notation by using the same symbol for a measure and its density; we will continue to do this. For each $j \in \mathbb{Z}_{\geq 0}$,

$$
\operatorname{supp}\left(\mu_{j}\right)=\bigcup_{a \in A_{j}}\left[a, a+N_{j}^{-1}\right] .
$$

Moreover, for every $j, k \in \mathbb{Z}_{\geq 0}$ with $j \leq k$ and for every $a \in A_{j}$,

$$
\mu_{k}\left(\left[a, a+N_{j}^{-1}\right]\right)=T_{j}^{-1} .
$$

Lemma 4.2. The sequence $\left(\mu_{j}\right)_{j=0}^{\infty}$ converges weakly (i.e., in distribution) to a probability measure $\mu$.

Proof. For each $j \in \mathbb{Z}_{>0}$, let $F_{j}$ be the cumulative distribution function of $\mu_{j}$, i.e., $F_{j}(t)=\mu_{j}((-\infty, t])$ for all $t \in \mathbb{R}$. Let $t \in \mathbb{R}$. If $t \leq \min A_{j}$, then $F_{j}(t)=$ $F_{j+1}(t)=0$. Now assume $t \geq \min A_{j}$. Let $a(t)$ be the unique element of $A_{j}$ such that $a(t) \leq t<a(t)+N^{-j}$. Since $F_{j}(a)=F_{j+1}(a)$ for each $a \in A_{j}$ and because of (4.3), we have

$$
\begin{aligned}
\left|F_{j+1}(t)-F_{j}(t)\right| & \leq\left|\mu_{j+1}((a(t), t])-\mu_{j}((a(t), t])\right| \\
& \leq \mu_{j+1}\left(\left[a(t), a(t)+N_{j}^{-1}\right]\right)+\mu_{j}\left(\left[a(t), a(t)+N_{j}^{-1}\right]\right) \\
& =2 T_{j}^{-1} \approx N_{j}^{-\alpha} \leq 2^{-j \alpha} .
\end{aligned}
$$

It follows that $\left(F_{j}\right)_{j=0}^{\infty}$ is a uniformly Cauchy and (consequently) uniformly convergent sequence of continuous cumulative distribution functions. Therefore the limit $F$ is a continuous cumulative distribution function of a Borel probability measure $\mu$ on $\mathbb{R}$. Hence $\left(\mu_{j}\right)_{j=0}^{\infty}$ converges weakly to $\mu$. 
Combining (4.3) with Lemma 4.2 gives, for every $j \in \mathbb{Z}_{\geq 0}$ and $a \in A_{j}$,

$$
\mu\left(\left[a, a+N_{j}^{-1}\right]\right)=T_{j}^{-1} .
$$

Lemma 4.3. The support of $\mu$ is

$$
\operatorname{supp}(\mu)=\bigcap_{j=0}^{\infty} \operatorname{supp}\left(\mu_{j}\right)=\bigcap_{j=0}^{\infty} \bigcup_{a \in A_{j}}\left[a, a+N_{j}^{-1}\right] .
$$

Proof. The second equality is immediate from (4.2). For the first equality, we consider $\subseteq$ and $\supseteq$ separately.

$\subseteq$ : We prove the contrapositive. Suppose $x \in \mathbb{R} \backslash \operatorname{supp}\left(\mu_{j_{0}}\right)$ for some $j_{0} \in$ $\mathbb{Z}_{\geq 0}$. Since $\mathbb{R} \backslash \operatorname{supp}\left(\mu_{j_{0}}\right)$ is open, there is an open ball $B\left(x, r_{0}\right)$ contained in $\mathbb{R} \backslash \operatorname{supp}\left(\mu_{j_{0}}\right)$. Since $\left(\operatorname{supp}\left(\mu_{j}\right)\right)_{j=0}^{\infty}$ is a decreasing sequence of sets, $B\left(x, r_{0}\right)$ is contained in $\mathbb{R} \backslash \operatorname{supp}\left(\mu_{j}\right)$ for every $j \geq j_{0}$. Thus $\mu_{j}\left(B\left(x, r_{0}\right)\right)=0$ for every $j \geq j_{0}$. Choose a continuous function $\phi: \mathbb{R} \rightarrow \mathbb{R}$ such that $\mathbf{1}_{B\left(x, r_{0} / 2\right)} \leq \phi \leq \mathbf{1}_{B\left(x, r_{0}\right)}$. Then, since $\mu_{j} \rightarrow \mu$ weakly, we have $\mu\left(B\left(x, r_{0} / 2\right)\right) \leq \int \phi d \mu=\lim _{j \rightarrow \infty} \int \phi d \mu_{j} \leq$ $\lim _{j \rightarrow \infty} \mu_{j}\left(B\left(x, r_{0}\right)\right)=0$. So $\mu\left(B\left(x, r_{0} / 2\right)\right)=0$. It follows that $x \in \mathbb{R} \backslash \operatorname{supp}(\mu)$.

$\supseteq$ : Let $x \in \bigcap_{j=0}^{\infty} \operatorname{supp}\left(\mu_{j}\right)$. Let $B(x, r)$ be any open ball centered at $x$. Choose $j$ large enough that $N_{j}^{-1}<r / 2$. Since $x \in \operatorname{supp}\left(\mu_{j}\right)$, we have $\mu_{j}(B(x, r / 2))>0$, hence $B(x, r / 2)$ intersects $\left[a, a+N_{j}^{-1}\right]$ for some $a \in A_{j}$. Therefore $B(x, r)$ contains $\left[a, a+N_{j}^{-1}\right]$. Choose a continuous function $\phi: \mathbb{R} \rightarrow \mathbb{R}$ such that $\mathbf{1}_{\left[a, a+N_{j}^{-1}\right]} \leq$ $\phi \leq \mathbf{1}_{B(x, r)}$. By (4.3), $T_{j}^{-1}=\mu_{k}\left(\left[a, a+N_{j}^{-1}\right]\right) \leq \int \phi d \mu_{k}$ for all $k \geq j$. Then, since $\mu_{k} \rightarrow \mu$ weakly, we have $T_{j}^{-1} \leq \lim _{k \rightarrow \infty} \int \phi d \mu_{k}=\int \phi d \mu \leq \mu(B(x, r))$. So $\mu(B(x, r))>0$. Since $B(x, r)$ was arbitrary, it follows that $x \in \operatorname{supp}(\mu)$.

The measure $\mu$ is the so-called natural measure on the Cantor set (4.1).

Lemma 4.4. For every interval $I$ with diameter $|I|, \mu(I) \lesssim|I|^{\alpha}$.

Proof. If $|I|>1$, then

$$
\mu(I) \leq \mu(\mathbb{R})=1 \leq|I|^{\alpha}
$$

since $\mu$ is a probability measure. Now suppose $|I| \leq 1$. Choose $j_{0} \in \mathbb{Z}_{\geq 0}$ such that $N_{j_{+}}^{-1} \leq|I| \leq N_{j_{0}}^{-1}$. Assume $I$ intersects $\operatorname{supp}(\mu)$ (otherwise $\mu(I)=0$ ). Then $I$ intersects an interval $\left[a, a+N_{j_{0}}^{-1}\right]$ for some $a \in A_{j_{0}}$. Since $|I| \leq N_{j_{0}}^{-1}$ and $A_{j} \subseteq N_{j_{0}}^{-1}\left[N_{j_{0}}\right]$, there are at most two such intervals; call them $J_{1}$ and $J_{2}$. By (4.4), the choice of the sequences $\left(n_{j}\right)$ and $\left(t_{j}\right)$, and the choice of $j_{0}$, we have

$$
\mu(I)=\mu\left(I \cap J_{1}\right)+\mu\left(I \cap J_{2}\right) \leq \mu\left(J_{1}\right)+\mu\left(J_{2}\right)=\frac{2}{T_{j_{0}}} \approx \frac{1}{N_{j_{0}+1}^{\alpha}} \leq|I|^{\alpha}
$$

Lemma 4.5. For every interval $I$ with center in $E$ and diameter $|I| \leq 1, \mu(I) \gtrsim$ $|I|^{\alpha}$.

Proof. Choose $j_{0} \in \mathbb{Z}_{>0}$ such that $2 N_{j_{0}+1}^{-1} \leq|I| \leq 2 N_{j_{0}}^{-1}$. Since the center of $I$ belongs to $E=\bigcap_{j=0}^{\infty} \bigcup_{a \in A_{j}}\left[a, a+N_{j}^{-1}\right]$, it belongs to the interval $\left[a, a+N_{j_{0}+1}^{-1}\right]$ for some $a \in A_{j_{0}+1}$. Then, since $2 N_{j_{0}+1}^{-1} \leq|I|,\left[a, a+N_{j_{0}+1}^{-1}\right] \subseteq I$. By (4.4), the choice of the sequences $\left(n_{j}\right)$ and $\left(t_{j}\right)$, and the choice of $j_{0}$, we have

$$
\mu(I) \geq \mu\left(\left[a, a+N_{j_{0}+1}^{-1}\right]\right)=\frac{1}{T_{j_{0}+1}} \approx \frac{1}{N_{j_{0}+1}^{\alpha}}=\frac{1}{2^{\alpha} n_{j_{0}+1}^{\alpha}} \frac{2^{\alpha}}{N_{j_{0}}^{\alpha}} \geq \frac{1}{2^{\alpha} n_{*}^{\alpha}}|I|^{\alpha} .
$$


4.2. Proof of Theorem 4.1; Fourier Decay. We will now prove that the sets $B_{j+1, a}$ can be chosen so that (c) and (d) of Theorem 4.1 hold. Before beginning, we outline the proof and make two remarks.

The idea of the proof is to choose the sets $B_{j+1, a}$ using the probabilistic method. More specifically, for a given $j$ and a given set $A_{j}$ of numbers $a$, we choose each $B_{j+1, a}$ uniformly at random from a finite collection of possible sets. Thus the differences (i) $\widehat{\mu_{j+1}}(\xi)-\widehat{\mu_{j}}(\xi)$ and (ii) $\widehat{\mu_{j+1} \cdot \nu}(\xi)-\widehat{\mu_{j} \cdot \nu}(\xi)$ can be written as sums of finitely many independent random variables. We use Hoeffding's large deviation inequality to show that, with positive probability, the differences (i) and (ii) are small. We deduce that there exists a choice of the sets $B_{j+1, a}$ which makes (i) and (ii) small. This probabilistic argument is the heart of the proof. It appears as Lemma 4.13 below. After Lemma 4.13, we use telescoping sum and geometric series arguments to deduce the desired decay estimates for $\widehat{\mu}$ and $\widehat{\mu \cdot \nu}$. Before getting to Lemma 4.13, we give several preparatory results.

Remark 4.6. The sets $B_{j+1, a}$ depend on $\nu$ because they are chosen (probabilistically) to make $\widehat{\mu_{j+1} \cdot \nu}(\xi)-\widehat{\mu_{j} \cdot \nu}(\xi)$ small. It would be possible to work with a finite or countably infinite collection of measures $\nu_{1}, \nu_{2}, \ldots$ and choose the sets $B_{j+1, a}$ so that $\widehat{\mu_{j+1} \cdot \nu_{i}}(\xi)-\widehat{\mu_{j} \cdot \nu_{i}}(\xi)$ is small for each $i$. (As an exercise, we invite the reader to verify this by modifying the proof of Lemma 4.13). Countable subadditivity of the probability measure is what makes this possible. However, we do not know how to choose the sets $B_{j+1, a}$ so that $\widehat{\mu_{j+1} \cdot \nu}(\xi)-\widehat{\mu_{j} \cdot \nu}(\xi)$ is small for all measures $\nu$. If we could do that, then we could make the measure $\mu$ independent of $\nu$ in Theorem 4.1, and hence make the set $Y$ independent of $R$ in Theorem 1.6.

Now we begin the proof. Let $j \in \mathbb{Z}_{>0}$. We write the densities of $\mu_{j}$ and $\mu_{j+1}$ in more convenient forms. By partitioning $\left[0, N_{j}^{-1}\right]$ into intervals of length $N_{j+1}^{-1}$, we see that

$$
\mu_{j}=\left(\frac{N_{j+1}}{T_{j}}\right)\left(\frac{1}{n_{j+1}}\right) \sum_{a \in A_{j}} \sum_{b \in N_{j+1}^{-1}\left[n_{j+1}\right]} \mathbf{1}_{a+b+\left[0, N_{j+1}^{-1}\right]} .
$$

Since $A_{j+1}=\bigcup_{a \in A_{j}}\left(a+B_{j+1, a}\right)$, we also have

$$
\mu_{j+1}=\left(\frac{N_{j+1}}{T_{j}}\right)\left(\frac{1}{t_{j+1}}\right) \sum_{a \in A_{j}} \sum_{b \in B_{j+1, a}} \mathbf{1}_{a+b+\left[0, N_{j+1}^{-1}\right]} .
$$

For each $a \in A_{j}, b \in B_{j+1, a}$, and $\xi \in \mathbb{R}$, define

$$
\begin{aligned}
I(a, b, j, \xi) & =\int_{[0,1]} e^{-2 \pi i\left(\xi / N_{j+1}\right)\left(a N_{j+1}+b N_{j+1}+x\right)} d x, \\
J(a, b, j, \xi) & =\int_{\mathbb{R}} \int_{[0,1]} e^{-2 \pi i\left(\xi / N_{j+1}\right)\left(a N_{j+1}+b N_{j+1}+x\right) y} d x d \nu(y), \\
X_{a}(j, \xi) & =\frac{1}{t_{j+1}} \sum_{b \in B_{j+1, a}} I(a, b, j, \xi)-\frac{1}{n_{j+1}} \sum_{b \in N_{j+1}^{-1}\left[n_{j+1}\right]} I(a, b, j, \xi), \\
Y_{a}(j, \xi) & =\frac{1}{t_{j+1}} \sum_{b \in B_{j+1, a}} J(a, b, j, \xi)-\frac{1}{n_{j+1}} \sum_{b \in N_{j+1}^{-1}\left[n_{j+1}\right]} J(a, b, j, \xi) .
\end{aligned}
$$


It follows that, for all $\xi \in \mathbb{R}$,

$$
\begin{aligned}
\widehat{\mu_{j+1}}(\xi)-\widehat{\mu_{j}}(\xi) & =\frac{1}{T_{j}} \sum_{a \in A_{j}} X_{a}(j, \xi) \\
\widehat{\mu_{j+1} \nu}(\xi)-\widehat{\mu_{j} \nu}(\xi) & =\frac{1}{T_{j}} \sum_{a \in A_{j}} Y_{a}(j, \xi) .
\end{aligned}
$$

By direct calculation, we have

Lemma 4.7. For each $j \in \mathbb{Z}_{j \geq 0}, a \in A_{j}, b \in B_{j+1, a}$, and $\xi \in \mathbb{R}$, we have

$$
\begin{aligned}
|I(a, b, j, \xi)| & \left.\leq \min \left\{1, N_{j+1} /|\xi|\right)\right\}, \\
\left|X_{a}(j, \xi)\right| & \left.\leq 2 \min \left\{1, N_{j+1} /|\xi|\right)\right\} .
\end{aligned}
$$

Define $g:[0, \infty) \rightarrow[0, \infty)$ by

$$
g(x)=(1+x)^{-1 / 2}+\sup \{|\widehat{\nu}(t x)|: t \in \mathbb{R},|t| \geq 1\}
$$

The following properties of $g$ are straightforward to verify.

\section{Lemma 4.8.}

(a) $g$ is non-increasing

(b) For all $\xi \in \mathbb{R},|\widehat{\nu}(\xi)| \leq g(|\xi|)$.

(c) For all $0 \leq \beta \leq 1, \sup _{\xi \in \mathbb{R}}|\widehat{\nu}(\xi)|(1+|\xi|)^{\beta / 2}<\infty$ if and only if $\sup _{\xi \in \mathbb{R}} g(|\xi|)(1+$ $|\xi|)^{\beta / 2}<\infty$.

Recall that $\operatorname{supp}(\nu)$ is compact and does not contain 0. Choose a Schwartz function $\phi: \mathbb{R} \rightarrow \mathbb{C}$ such that $\phi(y)=1 / y$ for all $y \in \operatorname{supp}(\nu)$.

Lemma 4.9. For all $x \in \mathbb{R},|(\widehat{\phi \nu})(x)| \lesssim g\left(\frac{1}{2}|x|\right)$.

Proof. Write

$$
|(\widehat{\phi \nu})(x)|=|(\widehat{\phi} * \widehat{\nu})(x)| \leq \int_{\mathbb{R}}|\widehat{\phi}(y)| \widehat{\nu}(|x-y|) d y .
$$

Bound the integral above by the sum of the integrals over $R_{1}=\left\{y: \frac{1}{2}|x| \leq|x-y|\right\}$ and $R_{2}=\left\{y: \frac{1}{2}|x| \leq|y|\right\}$. For the integral over $R_{1}$, use (a) and (b) of Lemma 4.8. For the integral over $R_{2}$, note $|\widehat{\phi}(y)|^{1 / 2} \lesssim g(|y|)$ for all $y \in \mathbb{R}$ (because $\widehat{\phi}$ is Schwartz), then use (a) of Lemma 4.8 .

Lemma 4.10. There is a constant $C_{0}>0$ such that for each $j \in \mathbb{Z}_{\geq 0}, a \in A_{j}$, $b \in B_{j+1, a}$, and $\xi \in \mathbb{R}$, we have

$$
\begin{aligned}
|J(a, b, j, \xi)| & \left.\leq C_{0} g\left(\frac{1}{2}|\xi|\right) \min \left\{1, N_{j+1} /|\xi|\right)\right\}, \\
\left|Y_{a}(j, \xi)\right| & \left.\leq 2 C_{0} g\left(\frac{1}{2}|\xi|\right) \min \left\{1, N_{j+1} /|\xi|\right)\right\} .
\end{aligned}
$$

Proof. Note (4.14) is immediate from (4.13). Integrating $y$ in (4.6) shows that

$$
J(a, b, j, \xi)=\int_{[0,1]} \widehat{\nu}\left(\left(\xi / N_{j+1}\right)\left(a N_{j+1}+b N_{j+1}+x\right)\right) d x
$$

Since $\left|\left(\xi / N_{j+1}\right)\left(a N_{j+1}+b N_{j+1}+x\right)\right| \geq|\xi|$ for each $x \in[0,1]$, (a) and (b) of Lemma 4.8 give

$$
|J(a, b, j, \xi)| \leq g(|\xi|)
$$


On the other hand, integrating $x$ in (4.6) shows that

$$
J(a, b, j, \xi)=\int_{\mathbb{R}} e^{-2 \pi i\left(\xi / N_{j+1}\right)\left(a N_{j+1}+b N_{j+1}\right) y} \frac{e^{-2 \pi i\left(\xi / N_{j+1}\right) y}-1}{-2 \pi i\left(\xi / N_{j+1}\right) y} d \nu(y) .
$$

After multiplying by $-2 \pi i\left(\xi / N_{j+1}\right)$, using that $\phi(y)=1 / y$ for all $y \in \operatorname{supp}(\nu)$, and integrating $y$, we see that $-2 \pi i\left(\xi / N_{j+1}\right) J(a, b, j, \xi)$ is

$$
=\widehat{\phi \nu}\left(\left(\xi / N_{j+1}\right)\left(a N_{j+1}+b N_{j+1}+1\right)\right)-\widehat{\phi \nu}\left(\left(\xi / N_{j+1}\right)\left(a N_{j+1}+b N_{j+1}\right)\right) .
$$

Since $\left|\left(\xi / N_{j+1}\right)\left(a N_{j+1}+b N_{j+1}+x\right)\right| \geq|\xi|$ for each $x \in[0,1]$, Lemma 4.9 and (a) of Lemma 4.8 give

$$
|J(a, b, j, \xi)| \lesssim \frac{1}{\pi}\left(\frac{N_{j+1}}{|\xi|}\right) g\left(\frac{1}{2}|\xi|\right)
$$

We need the following fact about averages over random subsets.

Lemma 4.11. Let $t \leq n$ be positive integers. Let $A$ be a finite set of size $n$, and let $F: A \rightarrow \mathbb{C}$. Let $\mathcal{B}_{t}$ be the collection of all size $t$ subsets of $A$, and let $B$ be a set chosen uniformly at random from $\mathcal{B}_{t}$. Then

$$
\mathbb{E}\left(\frac{1}{t} \sum_{x \in B} F(x)\right)=\frac{1}{n} \sum_{x \in A} F(x) .
$$

Proof. There are $\left(\begin{array}{l}n \\ t\end{array}\right)$ sets in $\mathcal{B}_{t}$. For each $x \in A$, there are $\left(\begin{array}{c}n-1 \\ t-1\end{array}\right)$ sets in $\mathcal{B}_{t}$ that contain $x$. Therefore

$\mathbb{E}\left(\frac{1}{t} \sum_{x \in B} F(x)\right)=\frac{1}{\left(\begin{array}{l}n \\ t\end{array}\right) \cdot t} \sum_{B \in \mathcal{B}_{t}} \sum_{x \in B} F(x)=\frac{1}{\left(\begin{array}{l}n \\ t\end{array}\right) \cdot t} \sum_{x \in A}\left(\begin{array}{c}n-1 \\ t-1\end{array}\right) F(x)=\frac{1}{n} \sum_{x \in A} F(x)$.

We need the following version of Hoeffding's inequality for complex-valued random variables.

Lemma 4.12. Suppose $Z_{1}, \ldots, Z_{t}$ are independent complex-valued random variables satisfying $\mathbb{E}\left(Z_{i}\right)=0$ and $\left|Z_{i}\right| \leq c$ for $i=1, \ldots, t$, where $c$ is a positive constant. For all $u>0$,

$$
\mathbb{P}\left(\left|\frac{1}{t} \sum_{i=1}^{t} Z_{i}\right| \geq c u\right) \leq 4 \exp \left(-\frac{1}{4} t u^{2}\right) .
$$

Proof. Apply the standard Hoeffding inequality to the real and imaginary parts of $Z_{1}, \ldots, Z_{t}$.

Lemma 4.13. Define $d_{0}$ by $1 / d_{0}=\max \{\operatorname{diam}(\operatorname{supp}(\mu)), \operatorname{diam}(\operatorname{supp}(\mu \cdot \nu))\}$. Fix a real number $\zeta_{0}$ such that

$$
\zeta_{0}>\sum_{k \in \mathbb{Z}} \frac{2}{1+d_{0}^{2}|k|^{2}} .
$$


It is possible to choose the sets $B_{j+1, a}$ such that, for every $j \in \mathbb{Z}_{\geq 0}$ and every $\xi \in d_{0} \mathbb{Z}=\left\{d_{0} k: k \in \mathbb{Z}\right\}$

$$
\left|\widehat{\mu_{j+1}}(\xi)-\widehat{\mu_{j}}(\xi)\right|<2 T_{j}^{-1 / 2} \ln ^{1 / 2}\left(4 \zeta_{0}\left(1+|\xi|^{2}\right)\right) \min \left\{1, N_{j+1} /|\xi|\right\},
$$

$$
\left|\widehat{\mu_{j+1} \nu}(\xi)-\widehat{\mu_{j} \nu}(\xi)\right|<2 C_{0} T_{j}^{-1 / 2} \ln ^{1 / 2}\left(4 \zeta_{0}\left(1+|\xi|^{2}\right)\right) g\left(\frac{1}{2}|\xi|\right) \min \left\{1, N_{j+1} /|\xi|\right\},
$$

where $C_{0}$ is the constant from Lemma 4.10.

Proof. Fix $j \in \mathbb{Z}_{\geq 0}$ and assume a set $A_{j} \subseteq[1,2)$ satisfying $\left|A_{j}\right|=T_{j}$ is given. To simplify notation in what follows, we write $N=N_{j+1}, n=n_{j+1}$, and $t=$ $t_{j+1}$. For each $a \in A_{j}$, suppose we choose $B_{j+1, a}$ independently and uniformly at random from the collection of all size $t$ subsets of $N^{-1}[n]$. Now fix $\xi \in \mathbb{R}$. Then $\left\{X_{a}(j, \xi): a \in A_{j}\right\}$ is a set of independent complex-valued random variables, and the same is true of $\left\{Y_{a}(j, \xi): a \in A_{j}\right\}$. Moreover, for each $a \in A_{j}$, we find that $\mathbb{E}\left(X_{a}(j, \xi)\right)=0$ by applying Lemma 4.11 with $F(b)=I(a, b, j, \xi)$ for $b \in$ $N^{-1}[n]$. Likewise, we find that $\mathbb{E}\left(Y_{a}(j, \xi)\right)=0$ by applying Lemma 4.11 with $F(b)=J(a, b, j, \xi)$. We also have the bounds on $\left|X_{a}(j, \xi)\right|$ and $\left|Y_{a}(j, \xi)\right|$ from Lemma 4.7 and Lemma 4.10, respectively. Define

$$
u_{j, \xi}=\sqrt{T_{j}^{-1} \ln \left(4 \zeta_{0}\left(1+|\xi|^{2}\right)\right)}
$$

Let $E^{1}(\xi)$ be the event that $\left|\frac{1}{T_{j}} \sum_{a \in A_{j}} X_{a}(j, \xi)\right|<2 u_{j, \xi} \min \{1, N /|\xi|\}$. Let $E^{2}(\xi)$ be the event that $\left|\frac{1}{T_{j}} \sum_{a \in A_{j}} Y_{a}(j, \xi)\right|<2 C_{0} u_{j, \xi} g\left(\frac{1}{2}|\xi|\right) \min \{1, N /|\xi|\}$. For each $\xi \in \mathbb{R}$, Lemma 4.12 implies

$$
\mathbb{P}\left(\left(E^{i}(\xi)\right)^{c}\right) \leq 4 \exp \left(-\frac{1}{4} T_{j} u_{j, \xi}^{2}\right)=\frac{1}{\zeta_{0}\left(1+|\xi|^{2}\right)} .
$$

Therefore, by De Morgan's laws and countable subaddivity, the probability that both $E^{1}\left(d_{0} k\right)$ and $E^{2}\left(d_{0} k\right)$ hold for all $k \in \mathbb{Z}$ is

$$
\geq 1-\sum_{k \in \mathbb{Z}}\left(\mathbb{P}\left(\left(E^{1}\left(d_{0} k\right)\right)^{c}\right)+\mathbb{P}\left(\left(E^{2}\left(d_{0} k\right)\right)^{c}\right)\right) \geq 1-\sum_{k \in \mathbb{Z}} \frac{2}{\zeta_{0}\left(1+d_{0}^{2}|k|^{2}\right)}>0 .
$$

In light of (4.9) and (4.10), this implies there is some choice of the sets $B_{j+1, a}$ $\left(a \in A_{j}\right)$ such that (4.15) and (4.16) hold for every $\xi \in d_{0} \mathbb{Z}$.

Lemma 4.14. With the sets $B_{j+1, a}$ chosen as in Lemma 4.13.

$$
\begin{aligned}
|\widehat{\mu}(\xi)| & \lesssim(1+|\xi|)^{-\alpha / 2} \ln ^{1 / 2}\left(4 \zeta_{0}\left(1+|\xi|^{2}\right)\right) \quad \forall \xi \in \mathbb{R}, \\
|\widehat{\mu \cdot \nu}(\xi)| & \lesssim(1+|\xi|)^{-\alpha / 2} g\left(\frac{1}{2}|\xi|\right) \ln ^{1 / 2}\left(4 \zeta_{0}\left(1+|\xi|^{2}\right)\right) \quad \forall \xi \in \mathbb{R} .
\end{aligned}
$$

Proof. We prove only (4.18), as the proof of (4.17) is similar and simpler. We begin by making two reductions.

First, by a standard argument (see Kahane [9, pp.252-253]), we only need to prove (4.18) for $\xi=d_{0} k \in d_{0} \mathbb{Z}$. For the second reduction, note that for every 
$0 \neq \xi \in \mathbb{R}$, we have

$$
\begin{aligned}
\widehat{\mu_{0} \cdot \nu}(\xi) & =\int_{\mathbb{R}} \int_{1}^{2} e^{-2 \pi i x y \xi} d x d \nu(y)=\int_{\mathbb{R}} \frac{e^{-2 \pi i y \xi}-e^{-2 \pi i y(2 \xi)}}{-2 \pi i y \xi} d \nu(y) \\
& =\int_{\mathbb{R}} \frac{e^{-2 \pi i y \xi}-e^{-2 \pi i y(2 \xi)}}{-2 \pi i \xi} \phi(y) d \nu(y)=\frac{1}{-2 \pi i \xi}(\widehat{\phi \nu}(\xi)-\widehat{\phi \nu}(2 \xi)) .
\end{aligned}
$$

By Lemma 4.9 and (a) of Lemma $4.8\left|\widehat{\mu_{0} \cdot \nu}(\xi)\right| \lesssim(1+|\xi|)^{-1} g\left(\frac{1}{2}|\xi|\right)$ for all $0 \neq \xi \in$ $\mathbb{R}$. The same inequality holds when $\xi=0$ by direct calculation. Therefore, by the triangle inequality, we just need to prove (4.18) with the left-hand side replaced by $\left|\widehat{\mu \cdot \nu}(\xi)-\widehat{\mu_{0} \cdot \nu}(\xi)\right|$.

Lemma4.2 says $\mu_{j} \rightarrow \mu$ weakly. Then the dominated convergence theorem shows that $\mu_{j} \cdot \nu \rightarrow \mu \cdot \nu$ weakly. Therefore, for every $\xi \in \mathbb{R}, \widehat{\mu \cdot \nu}(\xi)=\lim _{j \rightarrow \infty} \widehat{\mu_{j} \cdot \nu}(\xi)$, hence

$$
\left|\widehat{\mu \cdot \nu}(\xi)-\widehat{\mu_{0} \cdot \nu}(\xi)\right| \leq \sum_{j=0}^{\infty}\left|\widehat{\mu_{j+1} \cdot \nu}(\xi)-\widehat{\mu_{j} \cdot \nu}(\xi)\right| .
$$

If $\xi=0$, each term of the sum in 4.19) is zero, by direct calculation. Now assume $\xi=d_{0} k \in d_{0} \mathbb{Z}, \xi \neq 0$. By Lemma 4.13, the sum in (4.19) is

$$
\leq 2 C_{0} g\left(\frac{1}{2}|\xi|\right) \ln ^{1 / 2}\left(4 \zeta_{0}\left(1+|\xi|^{2}\right)\right)\left(\sum_{j: N_{j+1}>|\xi|} T_{j}^{-1 / 2}+\sum_{j: N_{j+1} \leq|\xi|} T_{j}^{-1 / 2} \frac{N_{j+1}}{|\xi|}\right) .
$$

To estimate the last two sums, recall that $2 \leq n_{j} \leq n_{*}, N_{j}=n_{1} \cdots n_{j}, N_{0}=$ $T_{0}=1$, and $T_{j} \approx N_{j}^{\alpha}$ for all $j \in \mathbb{Z}_{>0}$. Thus the first sum is

$$
\approx|\xi|^{-\alpha / 2} \sum_{j: N_{j+1}>|\xi|}\left(N_{j+1} /|\xi|\right)^{-\alpha / 2} \leq|\xi|^{-\alpha / 2} \sum_{k=0}^{\infty} 2^{-k \alpha / 2} \lesssim|\xi|^{-\alpha / 2} .
$$

And the second sum is

$$
\approx|\xi|^{-\alpha / 2} \sum_{j: N_{j+1} \leq|\xi|}\left(N_{j+1} /|\xi|\right)^{1-\alpha / 2} \leq|\xi|^{-\alpha / 2} \sum_{k=0}^{\infty} 2^{-k(1-\alpha / 2)} \lesssim|\xi|^{-\alpha / 2} .
$$

Lemma 4.15. With the sets $B_{j+1, a}$ chosen as in Lemma 4.13, $\operatorname{dim}_{F}(\mu) \geq \alpha$ and $\operatorname{dim}_{F}(\mu \cdot \nu) \geq \min \left\{1, \operatorname{dim}_{F}(\mu)+\operatorname{dim}_{F}(\nu)\right\}$.

Proof. Lemma 4.14 implies $\operatorname{dim}_{F}(\mu) \geq \alpha$. Lemma 4.14 and (c) of Lemma 4.8 imply $\operatorname{dim}_{F}(\mu \cdot \nu) \geq \operatorname{dim}_{F}(\mu)+\operatorname{dim}_{F}(\nu)$.

\section{REFERENCES}

1. J. Bourgain, Averages in the plane over convex curves and maximal operators, J. Analyse Math. 47 (1986), 69-85. MR 874045

2. 193-236. MR 2763000

3. X. Chen, Sets of Salem type and sharpness of the $L^{2}$-Fourier restriction theorem, Trans. Amer. Math. Soc. 368 (2016), no. 3, 1959-1977. MR 3449230

4. Kenneth Falconer, Fractal geometry, third ed., John Wiley \& Sons, Ltd., Chichester, 2014, Mathematical foundations and applications. MR 3236784

5. K. Hambrook, Restriction theorems and Salem sets, Ph.D. thesis, University of British Columbia, 2015. 
6. K. Hambrook and I. Laba, On the sharpness of Mockenhaupt's restriction theorem, Geom. Funct. Anal. 23 (2013), no. 4, 1262-1277. MR 3077913

7. Kyle Hambrook and Izabella Łaba, Sharpness of the Mockenhaupt-Mitsis-Bak-Seeger restriction theorem in higher dimensions, Bull. Lond. Math. Soc. 48 (2016), no. 5, 757-770. MR 3556359

8. A. Iosevich, personal communication.

9. J.-P. Kahane, Some random series of functions, second ed., Cambridge Studies in Advanced Mathematics, vol. 5, Cambridge University Press, Cambridge, 1985. MR 833073

10. B. Krause, A. Iosevich, E. Sawyer, K. Taylor, and I. Uriarte-Tuero, Maximal operators: scales, curvature and the fractal dimension, to appear in Analysis Mathematica, 2018.

11. Izabella Łaba and Malabika Pramanik, Arithmetic progressions in sets of fractional dimension, Geom. Funct. Anal. 19 (2009), no. 2, 429-456. MR 2545245

12. J. M. Marstrand, Packing circles in the plane, Proc. London Math. Soc. (3) 55 (1987), no. 1, 37-58. MR 887283

13. P. Mattila, Spherical averages of Fourier transforms of measures with finite energy; dimension of intersections and distance sets, Mathematika 34 (1987), no. 2, 207-228. MR 933500

14. $\ldots$ Geometry of sets and measures in Euclidean spaces, Cambridge Studies in Advanced Mathematics, vol. 44, Cambridge University Press, Cambridge, 1995, Fractals and rectifiability. MR 1333890

15. - Fourier analysis and Hausdorff dimension, Cambridge Studies in Advanced Mathematics, vol. 150, Cambridge University Press, Cambridge, 2015. MR 3617376

16. T. Mitsis, On a problem in geometric measure theory related to sphere and circle packing, ProQuest LLC, Ann Arbor, MI, 1998, Thesis (Ph.D.)-California Institute of Technology. MR 2697868

17. G. Mockenhaupt, Salem sets and restriction properties of Fourier transforms, Geom. Funct. Anal. 10 (2000), no. 6, 1579-1587. MR 1810754

18. D. M. Oberlin, Packing spheres and fractal Strichartz estimates in $\mathbb{R}^{d}$ for $d \geq 3$, Proc. Amer. Math. Soc. 134 (2006), no. 11, 3201-3209. MR 2231903

19. U Unions of hyperplanes, unions of spheres, and some related estimates, Illinois J. Math. 51 (2007), no. 4, 1265-1274. MR 2417426

20. K. Simon and K. Taylor, Dimension and measure of sums of planar sets and curves, arXiv:1706.00131, 2017.

21. - Interior of sums of planar sets and curves, to appear in Proc. Cambridge Philos. Soc., 2018.

22. E. M. Stein, Maximal functions. I. Spherical means, Proc. Nat. Acad. Sci. U.S.A. 73 (1976), no. $7,2174-2175$. MR 420116

23. T. Wolff, A Kakeya-type problem for circles, Amer. J. Math. 119 (1997), no. 5, 985-1026. MR 1473067

24. L Local smoothing type estimates on $L^{p}$ for large p, Geom. Funct. Anal. 10 (2000), no. 5, 1237-1288. MR 1800068

25. T. H. Wolff, Lectures on harmonic analysis, University Lecture Series, vol. 29, American Mathematical Society, Providence, RI, 2003, With a foreword by Charles Fefferman and a preface by Izabella Łaba, Edited by Łaba and Carol Shubin. MR 2003254

San Jose State University, One Washington Square, San Jose, CA 95192

The Ohio State University, Columbus, OH 43210 